\title{
Use of Fast Transfer Analysis Cartridges for Cervical Sampling and Real Time PCR Based High Risk HPV Testing in Cervical Cancer Prevention - a Feasibility Study from South India
}

\author{
Ramshankar Vijayalakshmi ${ }^{1 *}$, Thangaraj Soundara Viveka ${ }^{1}$, JS Malliga ${ }^{1}$, \\ Kothandaraman Murugan ${ }^{2}$, Albert Kanchana ${ }^{1}$, Krishnamurthy Arvind ${ }^{3}$
}

\begin{abstract}
Background: Molecular testing for human papillomavirus (HPV) is the most objective and reproducible of all cervical cancer screening tests and also less demanding in terms of training and quality assurance. However, there is an impending need for cost effective molecular HPV testing methods with sampling ease, easy storage measures and minimum turn around times suitable for a low resource setting. Objective : Our aim was to evaluate the feasibility of using a fast transfer analysis (FTA) mini elute cartridge for cervical sampling to identify high risk HPV by real time PCR and to compare molecular HPV testing and Pap cytology testing to predict histologically confirmed cervical precancer (CIN 2+ lesions) in a cervical cancer prevention program. Materials and Methods: This was conducted as a pilot study $(n=200)$ on women sampled using FTA mini elute cartridges, genotyped by two different real time PCR assays, detecting 13 high risk HPV (HR HPV) species, including HPV16 along with its physical DNA status. Results obtained from each of the tests were compared and analysed using suitable statistical tests. Results: With FTA mini elute cartridge samples HR HPV positivity was seen in 48/200 (24\%). Of these, presence of HPV 16 DNA was observed in 28/48 (58.3\%) women. High risk HPV was positive in $20 \%(37 / 185)$ of women with benign cytology and $73.3 \%(11 / 15)$ of women with abnormal cytology findings. A very significant correlation $(\chi 2=22.090 ; p=0.000)$ was observed between cytology and HR HPV findings showing an increasing trend of HR HPV prevalence in $50 \%$ (1/2) of LSIL, $75 \%$ (3/4) of HSIL and $100 \%(3 / 3)$ of SCC. Of the CIN 2+ lesions identified by histopathology, 88.9\% (8/9) had HR HPV. A significant association $(\chi 2=11.223 ; p=0.001)$ of HR HPV and histopathologically confirmed CIN $2+$ lesions was found. Sensitivity of the two tests were comparable but specificity of Pap testing was better $(90.7 \%$ vs $70.4 \%)$ to predict histopathologically diagnosed cervical precancers. Conclusions: The current study explored the feasibility of using a FTA mini elute cartridge for cervical sampling for the first time in India as a part of a community based cervical cancer prevention program. We suggest that FTA based sampling is suitable and feasible for real time based HPV testing. Molecular HR HPV testing can be more sensitive and useful to identify high risk women requiring Pap testing which is more specific to detect histologically confirmed cervical precancer.
\end{abstract}

Keywords: Fast transfer analysis cartridge - high risk HPV - Pap test - cervical pre-cancers - screening prevention

Asian Pac J Cancer Prev, 16 (14), 5993-5999

\section{Introduction}

Cervical cancer is the fourth most common cancer in women worldwide. Most importantly majority of cervical cancer deaths occur in less developed regions. In India, cervical cancer constitutes $27.4 \%$ of all incident cancers among women, with 308,901 new cases in 2014. The predicted estimates of mortality due to cervical cancer among Indian women by 2020 is 60,423 for women $<65$ years of age and 22,947 for women greater than or equal to 65 years of age (Ferlay et al., 2012). The aim of cervical cancer screening is to identify and treat precancerous lesions before they become invasive. The high burden of cervical cancer in developing countries is largely due to a lack of screening that allows detection of precancerous and early stage cervical cancer (Jemal et al., 2011). Previous studies have shown benefits of screening in women of low socioeconomic status and younger age more than those of high socioeconomic status and older age (Thulaseedharan et al., 2013)

HPV molecular test has been suggested as a primary screen to be used in conjunction with cytological screening approaches due to two different observations, HPV testing is $25 \%$ more sensitive but has $10 \%$ lower specificity. Secondly, a combination of HPV testing with cytology gives a negative predictive value of nearly

${ }^{I}$ Department of Preventive Oncology, ${ }^{2}$ Department of Cytology, ${ }^{3}$ Department of Surgical Oncology, Cancer Institute (WIA), Gandhi Nagar, Adyar, Chennai, India*For correspondence: vijiciwia@gmail.com 
$100 \%$ (Monsonego et al., 2004). Several recent studies show strengthened evidence supporting high risk human papillomavirus (HR HPV) testing as primary testing very substantially. (Gyllensten et al., 2012; Leinonen et al., 2012; Malila et al., 2012; Oglivie et al., 2012; RijKaart et al., 2012; Ronco et al., 2014).

However, for a low resource country like India, HPV testing using Hybrid Capture 2 (HC2), which is a widely used FDA approved test worldwide, may not be a very cost effective option (Pimple et al., 2014). Therefore there is an impending need for cost effective alternatives for performing HR HPV testing with sampling ease, convenient and suitable storage options, and also minimum turnaround times for determining HR HPV prevalence.

In the current study we have explored the feasibility of using a fast transfer analysis - (FTA) mini elute cartridge for cervical sampling for the first time in India as a part of an ongoing community based cervical cancer prevention program. FTA mini elute cartridge is a solid carrier device, with a patented technology that lyses the cells soon after sampling and prevents infection from samples. These are light, can be sampled and shipped at room temperatures and therefore suitable for Indian tropical conditions in a community based setting. HR HPV testing was done using real time PCR assays to detect 13 different HR HPV types as a single tube reaction as well as a HPV 16 specific assay to determine the prevalence and the physical status of the HPV DNA.

\section{Materials and Methods}

The current study is a part of Institution Ethics approved ongoing community based cervical cancer screening program in Kanchipuram District, in Tambaram and Chengelpet Taluk. Household survey was done and eligible women who consented to screen themselves were interviewed and invited to be a part of the screening. A Medical Social Worker or a trained health worker distributed the written subject information material in local language explaining the nature and the purpose of the study. Subject information material and informed consent forms were read out to the subject participants where ever necessary before obtaining signature or left thumb impression. A detailed socio-demographic and reproductive history was then obtained by the health workers in a structured questionnaire before subjecting them to screening. Ethical guidelines in terms of subject confidentiality was strictly maintained for the test results and the subjects with abnormal results were informed and treated in the Gynecologic Clinic free of cost.

\section{FTA based Sampling}

All the women $(n=200)$ were sampled for HPV testing using the FTA mini Elute cartidge (GE Healthcare, Sweden). The cartridges were air dried and transported to the molecular research laboratory from the community for testing.

Visual Inspection with Acetic Acid and Lugol's Iodine: All the subjects who consented for screening $(n=200)$ were subjected to cervix examination with $3-5 \%$ acetic acid and Lugol's iodine and evaluated for acetowhite epithelial changes by trained health workers. Women with suspicious acetowhite findings were posted for colposcopy and biopsy was taken from the suspicious lesions.

\section{Processing for HR HPV testing}

Five punches were made from each of the FTA mini elute cartridge and DNA was eluted out as per the manufacturer's instructions. DNA obtained was quantified using Biophotometer (Eppendorf, Hamburg, Germany) and used for quality control PCR amplifying beta globin gene using primers and conditions as mentioned before (Ramshankar et al., 2014). Samples positive for $\beta$ globin were taken for HR HPV testing using real time PCR.

\section{HPV Genotyping Protocol}

HPV Genotyping was performed using the 13 Highrisk HPV Real-time PCR kit (HBRT-H13C, Hybribio Limited , Hong Kong) according to the manufacturer's protocol. According to the manufacturers, this kit can detect presence of 13 high risk HPV $(16,18,31,33,35,39$, $45,51,52,56,58,59$ and 68 DNA in a single tube reaction using Real time PCR. DNA obtained from the samples collected was lysed with cell lysis solution provided with the kit. PCR was performed with a reaction volume of 20 $\mu \mathrm{l}$ containing $2 \mu \mathrm{l}$ of DNA template, $17.5 \mu \mathrm{l}$ of the master mixture provided, and $0.5 \mu \mathrm{l}$ of DNA Taq polymerase in a 7500 real-time PCR System (Applied Biosystems, Invitrogen, Carlsbad, CA, USA). The amplification protocol was as follows: $60^{\circ} \mathrm{C}$ for 1 minute, 10 minutes of denaturation at $95^{\circ} \mathrm{C}$ and 45 cycles of 10 seconds of denaturation at $95^{\circ} \mathrm{C}, 50$ seconds of annealing at $58^{\circ} \mathrm{C}$ and 10 seconds at $38^{\circ} \mathrm{C}$. The positive and negative controls provided with the kit were included in each set of PCRs to assess the performance of the test. All the samples were tested for HPV 16 using a TaqMan-based 5' exonuclease quantitative real-time PCR assay described previously (Ramshankar et al., 2014). $2 \mu$ l of genomic DNA was used to set up a $20 \mu \mathrm{l}$ PCR reaction with $1 \mathrm{X}$ TaqMan Mastermix (Applied Biosystems, Invitrogen, Carlsbad, CA, USA), $100 \mathrm{nM}$ each of dual-labelled E2 and E6 probe and 100 $\mathrm{nM}$ of E2 and 75nM of E6 primers (Shrimpex Biotech Services Ltd., Chennai, India). Threshold cycle numbers $(\mathrm{Ct})$ were determined with 7500 real-time PCR System (Applied Biosystems, Invitrogen, Carlsbad, CA, USA). Genomic DNA from CaSki and SiHa cervical cancer cell lines were used as positive control. HeLa cell line genomic DNA which contains HPV18 was used as negative control for HPV 16 E6. Amplification of E6 in samples indicated the presence of HPV16. The ratio of E2 and E6 ORF was calculated to assess the physical status of DNA.

\section{Cytology and Histology}

Cytology by Pap testing was done on all samples $(n=200)$ with results of HR HPV status blinded and reported according to Bethesda nomenclature (Solomon et al., 2002) A threshold of greater than or equal to ASCUS was used to define abnormal cytology. The slides showing abnormal cytology classified as ASCUS, low grade squamous intraepithelial lesion (LSIL) or high grade squamous intraepithelial lesion (HSIL) and reviewed by 
Fast Transfer Analysis Cartridges for High Risk HPV Detection in Cervical Cancer Prevention

an board-certified cytotechnologist for final classification. Colposcopy was carried out by a trained gynecologist and was administered to all the subject participants tested screening positive for VIA. The colposcopic findings were classified into the following categories as (1) normal, (2) squamous metaplasia, (3) condyloma, (4) probably low grade lesion/cervical intraepithelial neoplasia (CIN-I) (5) probably high grade lesion/CIN2-3 and (6) invasive carcinoma.

Punch biopsy was obtained for abnormal colposcopy test result and the specimens were fixed in formalin and processed and reported using the CIN system by the histopathology. The reference standard for final diagnosis was determined by upto 2 experienced pathologists who were blinded to the study including demographic and laboratory data. True disease status was defined as CIN-II and worse lesions (CIN2+lesions)

Statistical Analysis

Subject data was entered using the EPIINFO software. Statistical Software SPSS version 16 was used for data analysis. The association between demographic factors and different tests were evaluated by $\chi 2$ tests of significance. Odds ratio and $95 \%$ Confidence intervals were evaluated and $\mathrm{p}$ value less than 0.05 was considered significant. Sensitivity, specificity and predictive values and their 95\% confidence intervals $(\mathrm{CI})$ for the different tests were calculated using $2 \times 2$ tables and standard formula.

\section{Results}

Table 1 shows the test results of VIA, Pap and Histopathology results categorised by HR HPV status

\section{VIA}

Abnormal acetowhite findings were observed by the healthworkers in 63/200 (31.5\%). These women were further evaluated by colposcopy.

\section{FTA based HR HPV testing}

Of the 200 samples collected using the FTA cartridge (Figure 1), $\beta$ globin was found to amplify from DNA obtained in all samples tested passing the quality control criteria. HR HPV positivity obtained by both the real time based assays was 48/200 (24\%), of which high risk HPV 16 was confirmed by the other assay in 28/48 (58.3\%). The physical status of HPV 16 DNA showed mixed forms of both episomal and integrated forms in $60.7 \%(17 / 28)$ and fully integrated status was observed in 39.2\%(11/28) women. Physical status of HPV 16 DNA did not have a correlation with the cytological abnormalities and histopathological results. High risk types other than HPV 16 was identified in 20/48 (41.66\%) samples. HPV 16 was identified in $7 / 159$ (4.4\%) which were found to be negatives tested by Hybribio method. The two methods of real time based assays had an agreement with Kappa Coeff of 0.531 .

\section{Cytology}

Of the 200 samples tested by Pap test, 185/200 (92.5\%) were found to be cytologically benign and 15 samples (7.5\%) had abnormal cytology (Table 1). Among the cytologically benign samples, 37/185 (20\%) were found to be HR HPV positive and 17/37 (45.9\%) were HPV 16 positive. A very significant correlation was observed between cytology and HR HPV findings $(\chi 2=22.090$; $\mathrm{p}=0.000$ ). There was an increasing trend of HR HPV prevalence with increasing epithelial abnormality as evaluated by Pap.

Interestingly, presence of HPV16 was $100 \%(6 / 6)$ among women in 41-50 years of age group with abnormal

Table 1. High Risk HPV Testing vs Cytology and Histopathology

\begin{tabular}{lrrr}
\hline & $\begin{array}{r}\text { HR HPV } \\
(\mathrm{n}=48)\end{array}$ & $\begin{array}{r}\text { HR HPV- } \\
(\mathrm{n}=152)\end{array}$ & \\
\hline VIA & & & \\
Positive $(\mathrm{N}=63)$ & $24(38 \%)$ & $39(61.9 \%)$ & $\mathrm{P}=0.002$ \\
Negative (137) & $24(17.5 \%)$ & $113(82.4 \%)$ & \\
Pap Test & & & \\
Pap Benign Cytology (N=185) & $37(20 \%)$ & $148(80 \%)$ & $\mathrm{P}=0.000$ \\
Pap Abnormal Cytology $(\mathrm{N}=15)$ & $11(73.33 \%)$ & $4(26.66 \%)$ & \\
Inconclusive & $2(100)$ & & \\
ASC-US (N=2) & $2(100 \%)$ & & \\
LSIL (N=2) & $1(50 \%)$ & $1(50 \%)$ & \\
HSIL (N=4) & $3(75 \%)$ & $1(25 \%)$ & \\
Malignant (N=3) & $3(100 \%)$ & & \\
Histopathology & $16(29 \%)$ & $38(70.3 \%)$ & $\mathrm{P}=0.001$ \\
Colposcopy Normal (54) & $8(88.8 \%)$ & $1(11.1 \%)$ & \\
Cervical Precancer - Cin2+ (N=9) & & & \\
\hline
\end{tabular}

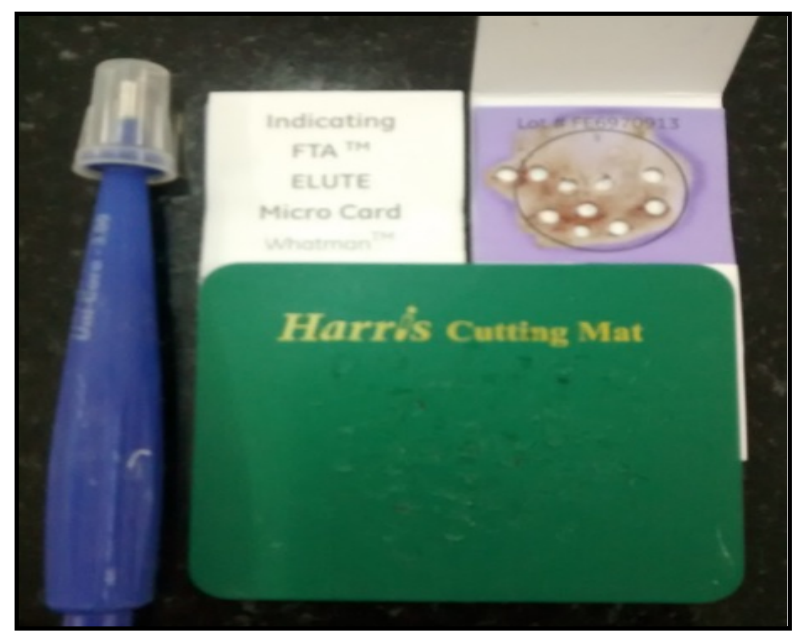

Figure 1. FTA Mini Elute Cartridge and Accessories

Table 2. Age vs Test Results

\begin{tabular}{|c|c|c|c|c|}
\hline & $\mathrm{N}$ & $\begin{array}{l}<40 \text { Years } \\
\text { Of Age }(\%)\end{array}$ & $\begin{array}{l}>40 \text { Years } \\
\text { Of Age }(\%)\end{array}$ & P Value \\
\hline \multicolumn{5}{|l|}{ Cytology Results } \\
\hline Normal & 185 & $116(62.7)$ & $69(37.2)$ & \\
\hline Abnormal & 15 & $5(33.3)$ & $10(66.6)$ & $\mathrm{P}=0.03$ \\
\hline \multicolumn{5}{|l|}{ Histology Results } \\
\hline BX Normal & 54 & $32(59.2)$ & $22(40.7)$ & \\
\hline BX-Cin 2+ & 9 & $2(22.2)$ & $7(77.7)$ & $\mathrm{P}=0.031$ \\
\hline \multicolumn{5}{|l|}{ HR HPV Results } \\
\hline HR HPV Positive & 48 & $27(56.25)$ & $21(43.75)$ & \\
\hline HR HPV Negative & 152 & $94(61.8)$ & $38.1)$ & \\
\hline \multicolumn{5}{|l|}{ VIA Results } \\
\hline VIA Positive & 63 & $37(58.7)$ & $26(41.26)$ & \\
\hline VIA Negative & 137 & $84(61.3)$ & $53(38.6)$ & \\
\hline
\end{tabular}


cytology results compared to $11.7 \%(2 / 52)$ in the same age group with benign cytology results, suggesting that presence of HPV 16 with increased age group should be closely monitored. This difference was statistically significant $\left(\chi^{2}=40.995, \mathrm{p}=0.000\right)$.

\section{Histology}

Abnormal histology was identified in 10 women of which 9/10 (90\%) were CIN 2+ lesions and one was identified as CIN I lesion. Of the 9 CIN2+ lesions, HR HPV was seen in $8 / 9(88.9 \%)$ which was a significant association $\left(\chi^{2}=12.228 ; \mathrm{p}=0.001\right)$ [Table 1]

\section{Age and test positives}

Median age of the women screened was 40 years with minimum age of 21 years and maximum of 59 years. Majority of the women 109/200 (54.5\%) belonged to age group 31-40 years, followed by 60/200 (30\%) in 41-50 years age group. Table 2 shows the test positives vs age group of participants. There was increasing abnormal cytology and CIN2+ findings with increasing age observed. Women above 40 years of age had $66.6 \%$ (10/15) and $85.1 \%$ (7/9) abnormal cytology and histology results respectively, compared to $43.75 \%$ (21/48) of HR HPV positive and $41.26 \%$ (26/63) and VIA positive in that age group. This suggests that presence of high risk HPV infection in women $>40$ years of age indicates closer monitoring and subjecting the women to pap testing, as they may be more likely to be at higher risk. If found abnormal by cytology testing, they should be posted for colposcopy examination to predict cervical precancers.

\section{Test Characteristics to detect CIN2+ lesions}

Table 3 shows the performance of HR HPV testing done from FTA elute cartridge based real time PCR to predict abnormal cytology. Sensitivity of HR HPV testing to predict an abnormal cytology was $73.3 \%$ (95\% CI $=44.90 \%-92.21 \%)$ ) and specificity was found to be 80\% (95\%CI=73.5\%-85.51\%) HR HPV testing had a Negative Predictive Value (NPV) of $97.37 \%$ (95\%CI ; 93.40\%-99.28\%) showing that a negative HR HPV result is meaningful to predict absence of the disease. Table 4 shows the sensitivity, specificity, positive likelihood ratio, negative likelihood ratio, positive predictive value (PPV) and NPV of the tests HR HPV and Pap test to predict CIN2+ lesions. Pap testing had a specificity of $90.74 \%$ (95\%CI ; 79.70\% - 96.92\%) compared to HR HPV testing with $70.37 \%$ specificity $(95 \% \mathrm{CI} ; 56.39 \%-82.02 \%)$. The sensitivity of the two tests were similar ; 88.89\% (51.75\%$99.72 \%$ ) to predict CIN2+. The Negative Predictive value (NPV) was comparable for both HR HPV and Pap testing.

\section{Discussion}

The current study shows cervical sampling using the FTA mini elute cartridges for HR HPV testing for the the first time in a community based setting in India and compares the performance of the molecular HPV test with conventional pap to predict histologically confirmed cervical pre-cancers. Use of FTA cartridges for HPV testing has been attempted before and shown the solid carrier as a promising device for collection of cervical specimens for screening, however, a highly sensitive method for HPV detection which are amplification based was suggested (Gustavsson et al., 2009; de Bie et al., 2011). This cartridge has been shown previously to be useful in tropical condition (Phongsavan et al., 2012). FTA mini elute cartridges involved no DNA extraction procedures saving cost and turnaround times suited for a high sample load and a low resource setting like ours. The housekeeping gene beta globin was found to amplify in all the samples showing that quality of DNA obtained from the mini elute cartridge to be suitable for PCR and other downstream applications. The cartridge offered ease of handling and storage for the trained healthworkers who were not very familiar with sampling for molecular testing.

There have been previous attempts to collect cervical scrapings on 3MM Whatman filter paper and used for HPV studies in India (Kailash et al., 2002). Dry paper smear technique has been shown recently in studies by Sarma et al., 2015. Our study has used a more improved version of the $3 \mathrm{MM}$ Whatman with a patented technology that prevents infection and followed it by a real time PCR based of 13 high risk subtypes in a single tube reaction saving time and labour with lesser turnaround times.

The updated and current guidelines for early detection of cervical cancer and its precursors recommend high risk HPV testing alone as a primary screening approach for cervical cancer as per American Cancer Society (ACS), the American society of colposcopy, and cervical pathology (ASCCP) and the American Society of Clinical Pathology (ASCP). These interim guidelines have been based on the guiding assumptions that no cancer screening test has the ability to detect all cases of prevalent or incipient

Table 3. Performance of HR HPV Testing to Predict Abnormal Cytology

\begin{tabular}{lcccccc}
\hline & Sensitivity & Specificity & Positive LR & Negative LR & PPV & NPV \\
\hline HR HPV & $73.33 \%$ & $80 \%$ & 3.67 & 0.33 & $22.92 \%$ & $97.37 \%$ \\
$95 \%$ CI & $44.90 \%-92.21 \%$ & $(73.5 \%-85.51 \%)$ & $(2.40-5.57)$ & $(0.14-0.77)$ & $(12.03 \%-37.31 \%)$ & $(93.40 \%-99.28 \%)$ \\
\hline
\end{tabular}

Table 4. Performance of HR HPV Testing and Pap Testing to Predict Cervical Precancer Lesions

\begin{tabular}{lcccccc}
\hline & Sensitivity & Specificity & Positive LR & Negative LR & PPV & NPV \\
\hline PAP TEST & $88.89 \%$ & $90.74 \%$ & 9.6 & 0.12 & $14.29 \%$ & $98 \%$ \\
95\% CI & $51.75 \%-99.72$ & $79.70-96.92$ & $4.04-22.83$ & $0.02-0.78$ & $6.75-25.39$ & $89.35-99.95$ \\
HR HPV TEST & $88.89 \%$ & $70.37 \%$ & 3 & 0.16 & 33.33 & 97.44 \\
$95 \%$ CI & $51.75 \%-99.72$ & $56.39 \%-82.02 \%$ & $1.87-4.81$ & $0.02-1.01$ & $15.63-55.32$ & $86.52-99.94$ \\
\hline
\end{tabular}


cervical cancer. Higher detection of CIN $3+$ at the baseline screening ground and reduced detection of CIN3+ at the subsequent screening rounds are considered as benefits. Also, increased number of colposcopies are considered a surrogate harms of screening (Monsonego et al., 2015). Studies show that a single HPV test at around 35 years of age is the most cost-effective intervention in reducing the burden of cervical cancer in countries with low or no screening coverage (Goldie et al., 2005). Women with atypical cells of undetermined significance (ASCUS) and LSIL on cervical cytology should be subjected to HPV DNA test and only if found positive should be referred for colposcopy as suggested by earlier studies (Katyal and Mehrotra 2011).

There are landmark studies conducted in India showing HPV testing as the most objective and reproducible of all cervical cancer screening tests and less demanding in terms of training and quality assurance (Huh et al., 2015). The argument for incorporating high risk HPV genotyping into screening strategies is supported by the finding that women with negative cytology who test positive for high risk HPV16/18 have a much greater short term risk of cervical precancer and long term risks of cervical cancer ( $\geq$ CIN3) compared to women who test positive for any of the 12 other HR-HPV types. (Khan et al., 2005, Wright et al., 2011, Cox et al., 2013). We had $20 \%$ of women harboring high risk HPV with benign cytology results and of these 9.2\% harbored HPV 16 infection, suggesting a closer monitoring for these women.

Our results showed a significant association between HR HPV prevalence and epithelial abnormalities detected in cervical smears very similar to earlier reports (Argyri et al., 2013; Sarma et al., 2015). The current study detected HPV 16 viral DNA in $50 \%$ of LSIL, $75 \%$ of HSIL and $100 \%$ of SCC. Women cytologically diagnosed as LSIL, AGUS and histologically found normal with high risk HPV 16 may have to be monitored based on the age group. We find that HR HPV infection increases over $50 \%$ with severe dyskaryosis seen in smears as shown previously (Kitcher et al., 2006). There are studies that show HR HPV infection in women $>30$ years of age, who have negative cytology findings having a higher risk conferred by these genotypes. This finding is more meaningful since HPV genotyping can be useful in triaging women with negative cytology to colposcopy. This study has shown that in women aged more than 30 years with negative cytology, subtypes HPV 16 and HPV18 conferred higher risk of CINIII (Kim et al., 2012). Therefore molecular HPV testing can help identify women at younger age and it would help to monitor these younger women for persistence in subsequent years.

One of the drawbacks of HR HPV testing is cost that is prohibitive for a low resource setting. FDA approved HC II has been used in several Indian studies (Sowjanya et al., 2005; Rai et al., 2014) and some studies have provided supporting evidence that self collected HC II have a similar sensitivity compared to the physician collected specimens (Bhatla et al, 2009; Sowjanya et al, 2009). However this assay is still very expensive and has been a major obstacle in its realization across the clinics of India.

We used FTA sampled, Hybribio real time PCR based approach to identify the 13 HR HPV types, which is a CE marked, SFDA approved kit for the current study. We have not been able to compare the performance of the $\mathrm{HC} 2$ and Hybribio in the current study. But earlier studies have shown that FTA eluted DNA have not suited HC2 protocol (de Bie et al., 2011). The current study test costed approximately $11 \$$ which is much cheaper than the FDA approved HC II done previously costing approximately $35 \$$ per test.

Another drawback in the community based screening programs other than cost perspective is the subject compliance. HR HPV testing needs repetition to evaluate the persistence of high risk viral infection in the population and repeat visits by women incurs additional cost of transport reducing compliance and leading to loss of follow up tests in the process of HR HPV testing. We hypothesise that use of FTA cartridges as a self sampling device can be tried and evaluated for improved subject compliance and cost effective measure.

Our studies showed that high risk HPV testing as a primary testing could predict eventually abnormal Pap result with a sensitivity of $73.3 \%$ and specificity of $80 \%$ with a NPV of $97.3 \%$. Comparing the Pap and HR HPV to predict an abnormal histology, Pap testing was found more specific with comparable sensitivity. There were $25.3 \%$ of women harbouring HR HPV and free of disease histopathologically. But these women warrant closer monitoring for persistence. The high negative predictive value (NPV) offered by HR HPV testing for an abnormal pap result and histologically confirmed precancer has been shown before (Lee et al., 2014). The HR-HPV testing may be therefore be useful for management of patients with abnormal cervical smears (Tao et al., 2014).

In the context of low resource setting, visual inspection with acetic acid (VIA) followed by treatment with cryotherapy has also been proposed as alternatives in cervical cancer, but studies evaluating VIA has been inconsistent (Sherigar et al., 2010; Parashari et al., 2013; Almonte et al., 2015) There are also studies showing VIA screening, in the presence of good training and sustained quality assurance, as an effective method to prevent cervical cancer in developing countries (Sankaranarayan et al., 2007). However, recent guidelines by WHO for screening and treatment of precancerous lesions in cervical cancer prevention proposes to use the HPV testing if available, followed by cryotherapy or by triage with VIA and then cryotherapy.

Our results showed a VIA positivity of $31.5 \%$ as screened by the health workers and $85.7 \%$ of these women were found to be normal after colposcopy however $80 \%$ of the abnormal histology findings were depicted as an abnormal VIA pattern of acetowhite staining. Some of the important causes of variations of sensitivity and specificity of VIA is suggested to be pertaining to man power training, variation of light source and procedure for preparation of $4-5 \%$ acetic acid, storage. (Parashari et al., 2013). An efficient quality assurance mechanism like access to expert opinion through immediate distance consultation as shown previously (Parham et al., 2010) will offer improvement.

In conclusion, the current pilot studies shows that FTA cartridge can be incorporated for cervical sampling 
in a routine cervical cancer prevention program. In combination with a low cost HR HPV genotyping as a single tube reaction it can be cost effective and also more suitable to repeated testing to assess the HR HPV persistence. It can additionally be evaluated for performance as a self sampling device in future.

\section{Acknowledgements}

We thank all the women who consented to be a part of this study, trained community healthworkers, who provided us with samples as a part of the community screening initiative. We thank Dr V Shanta, Chairperson, Cancer Institute and GE Healthcare, India for the funding and technical support.

\section{References}

Almonte M, Ferreccio C, Luciani S, et al. (2015) Visual inspection after acetic acid (VIA) is highly heterogeneous in primary cervical screening in Amazonian Peru. PLoS one, 10, 115355-67

Argyri E, Papaspyridakos S, Tsimplaki E, et al (2013). A cross sectional study of HPV type prevalence according to age and cytology. BMC Infect Dis, 13, 53-8.

Bhatla N, Dar L, Patro AR, et al (2009). Can human HPV in Healthy Women of North East India papillomavirus DNA testing of self-collected vaginal samples compare with physician-collected cervical samples and cytology for cervical cancer screening in developing countries? Cancer Epidemiol, 33, 446-50.

Cox JT, Castle PE, Behrens CM, et al (2013). Comparison of cervical cancer screening strategies incorporating different combinations of cytology, HPV testing, and genotyping for HPV 16/18: results from the ATHENA HPV study. Am $J$ Obstet Gynecol, 208, 184.

de Bie RP, Schmeink CE, Bakkers JM, et al (2011) The indicating FTA elute cartridge a solid sample carrier to detect highrisk HPV and high-grade cervical lesions. J Mol Diagn, 13, 371-6.

Ferlay J, Soerjomataram I, Ervik M, et al (2013). GLOBOCAN 2012 v1.0, Cancer Incidence and Mortality Worldwide: IARC Cancer Base No.11[Internet]. Lyon, France: International Agency for Research on Cancer.

Goldie SJ, Gaffikin L, Goldhaber-Fiebert JD, et al (2005). Cost effectiveness of cervical cancer screening in five developing countries. N Engl J Med, 353, 2158-68.

Gustavsson I, Lindell M, Wilander E, et al (2009) Use of FTA card for dry collection, transportation and storage of cervical cell specimen to detect high-risk HPV. J Clin Virol, 46, $112-116$

Gyllensten U, Gustavsson I, Lindell M, et al (2012). Primary high-risk HPV screening for cervical cancer in postmenopausal women. Gynecol Oncol, 125, 343-5

Huh WK, Ault KA, Chelmow D, et al (2015). Use of primary high-risk human papillomavirus testing for cervical cancer screening: Interim clinical guidance. Gynecol Oncol, 136, 178-82.

Jemal A, Bray F, Center MM, et al. Global cancer statistics (2011). CA Cancer J Clin, 61, 69-90

Kailash U, Hedau S, Gopalkrishna V, et al. (2002). A simple 'paper smear' method for dry collection, transport and storage of cervical cytological specimens for rapid screening of HPV infection by PCR. J Med Microbiol, 51, 606-10.

Katyal S, Mehrotra R (2011). Complementary procedures in cervical cancer screening in low resource settings. J Obstet Gynaecol India, 61, 436-8

Khan MJ, Castle PE, Lorincz AT, et al. (2005). The elevated 10 -year risk of cervical precancer and cancer in women with human papillomavirus (HPV) type 16 or 18 and the possible utility of type-specific HPV testing in clinical practice. J Natl Cancer Inst, 97, 1072-9.

Kim K1, Kim JJ, Kim SM, et al (2012). Prevalence and determinants of high-risk human papillomavirus infection in women with high socioeconomic status in Seoul, Republic of Korea. Asian Pac J Cancer Prev, 13, 269-73.

Kitchener HC, Almonte M, Thomson C et al (2009). HPV testing in combination with liquid-based cytology in primary cervical screening (ARTISTIC): a randomised controlled trial. Lancet Oncol, 10, 672-82.

Leinonen MK, Nieminen P, Lonnberg S, et al (2012). Detection rates of precancerous and cancerous cervical lesions within one screening round of primary human papillomavirus DNA testing: prospective randomised trial in Finland. $B M J, \mathbf{3 4 5}$, 77896-906.

Malila N, Leinonen M, Kotaniemi-Talonen L, et al (2013). The HPV test has similar sensitivity but more over diagnosis than the Pap test--a randomised health services study on cervical cancer screening in Finland. Int J Cancer, 132, 2141-7

Monsonego J1, (2004). Colposcopy: the value of HPV testing in clinical practice. Gynecol Obstet Fertil, 32, 62-74.

Monsonego J, Cox JT, Behrens C, et al (2015). Prevalence of high-risk human papilloma virus genotypes and associated risk of cervical precancerous lesions in a large U.S. screening population: Data from the ATHENA trial. Gynecol Oncol, 137, 47-54.

Ogilvie GS, Krajden M, van Niekerk DJ, et al (2012). Primary cervical cancer screening with HPV testing compared with liquid-based cytology: results of round 1 of a randomised controlled trial -- the HPV FOCAL Study. Br J Cancer, 107, 1917-24.

Parashari A, Singh V. (2013) Reasons for variation in sensitivity and specificity of visual inspection with acetic acid (VIA) for the detection of pre- cancer and cancer lesions of uterine cervix. Asian Pac J Cancer Prev, 14, 7761-2.

Parham GP, Mwanahamuntu MH, Pfaendler KS, et al (2010). eC3--a modern telecommunications matrix for cervical cancer prevention in Zambia. J Low Genit Tract Dis, 14, 167-73.

Pimple S, Shastri SS. (2014). Comparative evaluation of human papilloma virus-DNA test verses colposcopy as secondary cervical cancer screening test to triage screen positive women on primary screening by visual inspection with $5 \%$ acetic acid. Indian J Cancer, 5, 117-23.

Phongsavan K, Gustavsson I, Marions L, et al (2012). Detection of human papillomavirus among women in Laos: feasibility of using filter paper card and prevalence of high-risk types. Int J Gynecol Cancer, 22, 1398-406.

Rai AK, Das D, Kataki AC, et al. (2014). Hybrid capture 2 assay based evaluation of high-risk HPV status in healthy women of north-east India. Asian Pac J Cancer Prev, 15, 861-5.

Ramshankar V, Soundara VT, Shyamsundar V, et al. (2014). Risk stratification of early stage oral tongue cancers based on HPV status and p16 immunoexpression. Asian Pac J Cancer Prev, 15, 8351-9.

Rijkaart DC, Heideman DA, Coupe VM, et al (2012). High-risk human papillomavirus (hrHPV) E6/E7 mRNA testing by PreTect HPV-Proofer for detection of cervical high-grade intraepithelial neoplasia and cancer among hrHPV DNApositive women with normal cytology. J Clin Microbiol. 50, 2390-6.

Ronco G, Dillner J, Elfstrom KM, et al (2014). International HPV 
screening working group. Efficacy of HPV-based screening for prevention of invasive cervical cancer: follow-up of four European randomised controlled trials. Lancet, 383, 524-32

Sankaranarayanan R, Esmy PO, Rajkumar R, et al (2007) Effect of visual screening on cervical cancer incidence and mortality in Tamil Nadu, India: a cluster-randomised trial. Lancet, 370, 398-406.

Sarma U, Mahanta J, Borkakoty B, et al (2015). Distribution of human papilloma virus infections of uterine cervix among women of reproductive age--a cross sectional hospital-based study from North East India. Asian Pac J Cancer Prev, 16, 1519-23.

Sherigar B, Dalal A, Durdi G, et al (2010). Cervical cancer screening by visual Inspection with acetic acid - interobserver variability between nurse and physician. Asian Pac J Cancer Prev, 11, 619-22.

Solomon D, Davey D, Kurman R, et al. (2002). The 2001 Bethesda System: terminology for reporting results of cervical cytology. JAMA, 287, 2114-9.

Sowjanya AP, Jain M, Poli UR, et al (2005). Prevalence and distribution of high-risk human papilloma virus (HPV) types in invasive squamous cell carcinoma of the cervix and in normal women in Andhra Pradesh, India. BMC Infect Dis, 5, 116-23.

Sowjanya AP, Paul P, Vedantham H, et al (2009). Suitability of self-collected vaginal samples for cervical cancer screening in periurban villages in Andhra Pradesh, India. Cancer Epidemiol Biomarkers Prev, 18, 1373-8

Tao K, Yan J, Yang H, et al (2014). Comparative study of the Cervista and Hybrid Capture 2 methods in detecting high-risk human papillomavirus in cervical lesions. Diagn Cytopathology, 42, 213-7.

Thulaseedharan JV1, Malila N, Hakama M, et al (2013). Effect of screening on the risk estimates of socio demographic factors on cervical cancer- a large cohort study from rural India. Asian Pac J Cancer Prev, 14, 589-94.

Wright TC Jr, Stoler MH, Sharma A, et al (2011). Evaluation of HPV-16 and HPV-18 genotyping for the triage of women with high-risk HPV+ cytology-negative results. Am J Clin Pathol, 136, 578-86 